\title{
Risk management of emerging compounds and pathogens in the water cycle (RiSKWa)
}

\author{
Susanne Huckele ${ }^{*}$ and Thomas Track
}

\begin{abstract}
The increasing concentrations and diversity of trace pollutants and pathogens in the water cycle are a challenge with regard to their classification, assessment and elimination. As a result the Federal Ministry of Education and Research (BMBF) initiated the funding measure "Risk Management of Emerging Compounds and Pathogens in the Water Cycle (RiSKWa)". The aim of the programme is to develop innovative technologies and concepts for the risk management of these compounds towards a preventive environmental and health protection based on an application-oriented approach. Having started between October 2011 and January 2012, 12 joint research projects with more than 90 actors from science, industry, society and authorities are working on "Risk characterisation and management", "Technologies for emission and immission management" and "Measures for communication and education" during the next 3 years.
\end{abstract}

\section{Background}

The funding measure "Risk Management of Emerging Compounds and Pathogens in the Water Cycle (RiSKWa)" aims to develop an innovative and dynamic risk management system accompanied by on site demonstrations. In this framework the 12 joint research projects and the scientific support project of "RiSKWa" are working on the identification of risks as well as the development of technologies and strategies to avoid or reduce the appearance of emerging compounds and pathogens in the water cycle.

One of the central issues in RiSKWa is the evaluation of toxic relevant substances and thus evaluation concepts regarding eco-/human toxicology as well as microbiological aspects will be optimized and adapted. Concepts like inter alia REACH data or the healthrelated indication value (HRIV) concept are proven concepts for the identification and classification of emerging compounds and pathogens. Finally the results are relevant for drinking water supply, waste water disposal and water pollution control even if the requirements differ within the various sectors.

With regard to non-target screening a database to back up the gained data will be developed. In addition to

\footnotetext{
* Correspondence: huckele@dechema.de

DECHEMA Gesellschaft für Chemische Technik und Biotechnologie e. V., Theodor-Heuss-Allee-25, 60486, Frankfurt am Main, Germany
}

the identified substances their metabolites and resistances are also examined and documented. Due to little concentrations and a wide range of emerging compounds and pathogens the (further) development of established and new analytical methods is essential for a comprehensive risk management. Regarding drinking water supply existing installations are inspected and technologies to guarantee a high drinking water quality are optimized.

Impacts of climate change and demographic development cause increasing concentrations of anthropogenic trace substances and pose a challenge to urban water supply and water disposal in the medium to long term. To characterize the behavior of anthropogenic trace substances within the urban water cycle, knowledge on the impact of natural and technical barriers is essential. This issue is studied e.g. in Berlin and Dresden. In particular the emission of pharmaceuticals and pathogens from various health care facilities into the aquatic environment is an additional aspect which is investigated.

In rural areas diffuse pollution is the main source for emerging compounds in the water cycle. Persistence emanating from veterinary pharmacology micro pollutants and pathogens is investigated in a screening programme to determine their environmental impact. Exemplarily a process-based risk management of micropollutants and pathogens is evaluated for karstic aquifers at catchment scale on the Swabian Alb in Southwestern Germany. 
Major point sources for the release of micro pollutants and pathogens into the water cycle are sewage treatment plants. In order to minimize the impact due to discharging waster water into the water bodies and to guarantee a high water quality the implementation of an additional treatment process is being considered. Hence different technical approaches combining adsorptive, biological and oxidative waste water treatment are tested e.g. in the catchment areas of the rivers Schussen and Ruhr. The impact to the aquatic environment is observed in accompanying monitoring programmes.

Even so the 12 joint research projects have different priorities, in all of them training and communication measures will be elaborated in order to establish an effective risk management and a preventive environmental and health protection. The scientific support project provides an umbrella and communication platform for the joint research projects, acts as a first contact point for RiSKWa and represents the funding measure as a whole to the scientific community and the public.

\section{Joint research projects within the funding measure "Risk management of emerging compounds and pathogens in the water cycle (RiSKWa)"}

A main issue of the projects is the identification and classification of pollutants and pathogens relevant for the aquatic environment. An important aspect is the identification of new metabolites and resistances as well as their environmental impact.

Against this background the research project RISKIDENT develops and applies an identification system for both already known and so far unknown anthropogenic trace contaminants using LC-MS/MS. To back up the gained results of already known and so far unknown anthropogenic trace contaminants a database shall be developed named "STOFF-IDENT".

The aim is to bundle the existing information (e.g. $\mathrm{REACH}$ data) and the collected new information and data in one database and to provide these information to analytical laboratories to support the identification of unknown anthropogenic micro pollutants. The database "STOFF-IDENT" shall be linked with the database DAIOS. Besides the database a new method to eliminate trace contaminants during waste water treatment will be tested within RISK-IDENT. The project results will be summarized as guidelines on the identification and risk management of unknown trace contaminants, on procedures dealing with the elimination behaviour in sewage treatment plants and on ecotoxicological effects, mobility and relevance for drinking water resources.

The research projects PRiMaT, RiMaTH and ToxBox address risk management of emerging compounds and pathogens focusing on drinking water supplies.
For the production of safe and high quality drinking water the research project PRiMaT aims at the development of a risk-based master plan for handling trace pollutants and pathogens in drinking water supplies. The concept will be based on three pillars: risk analysis, risk mitigation and risk communication. Risk analysis will focus on a description of sources and propagation scenarios for emerging pollutants and pathogens in water catchment areas. Therefore molecular biological methods for microorganisms as well as concepts for the characterisation of trace pollutants, nano materials and pathogens will be developed and validated. With regard to risk mitigation technical solutions for raw water treatment as effective barrier especially against polar trace pollutants will be applied, combing innovative oxidation technologies with biological treatment steps. In addition novel adsorption-based and membrane-based technologies for selective removal of trace pollutants will be investigated. Risk communication will include educational measures and communication concepts for a balanced handling of risks from a water supplier's perspective. Furthermore a concept for educational measures at schools will be developed to increase consumer's awareness for drinking water issues in the long-term. Finally, examples from the past will be studied to learn about experiences on communication between water utilities and consumers and to identify areas for improvement.

The aim of the research project RiMaTH is to develop fast, miniaturized, chip-based methods for detection, classification and activity testing of relevant microorganisms (Legionella und Pseudomonas). These methods are embedded in a general concept of the evaluation of the sanitary situation as well as the risk management during decontamination. Therefore these chip-based molecularbiological methods will be implemented in addition to and evaluated against traditional culture techniques. In addition a proof-of-concept study on the use of Raman spectroscopy for pathogens detection will be implemented. These methods shall enable a pathogen classification at species level but also a live/dead discrimination. Furthermore, the sanitary situation of about 100 buildings with different designated uses is inspected within a monitoring programme to allow a comparison of the new approaches.

The evaluation of pollutants and pathogens for the protection of the drinking water supply is a central issue of the research project Tox-Box. The project aims at a harmonized testing strategy, laid down in a guideline, concerning an exposure-based and hazard-based risk management of anthropogenic trace substances. This approach is a key element of health related environmental policy. It is also reflected in current efforts to amend environmental regulations e.g. the European Water Framework Directive 
or the regulations on chemicals (REACH). The healthrelated indication value (HRIV concept) is used as the basis for risk assessment. Although this theoretical concept defines biological endpoints like genotoxicity, neurotoxicity, endocrine effects, germ cell damages, it does not devise the experimental approach, which is needed to obtain toxicological data. Decision trees as generic guidelines will be formulated including potential threats and cases requiring regulation, the determination of possible ways of action.

The emission of pollutants and pathogens due to urban areas are in the focus of ASKURIS for the City of Berlin and of ANTI-Resist for the City of Dresden. In particular sewage from health care facilities is characterized within the research project SAUBER+.

Due to the impact of climate change and demographic development, the concentrations of anthropogenic trace compounds are anticipated to increase especially in urban surface and ground waters, e.g. in Berlin. Based on this the research project ASKURIS aims at ensuring the drinking water supply of the City of Berlin. The emerging risks induced by anthropogenic trace substances and multi-resistant bacteria are characterized and assessed using analytical techniques in combination with effect-related assays. The reduction of anthropogenic trace substances and multi-resistant bacteria due to natural and technical barriers will be determined and investigated, e.g. the adsorption of organic compounds to activated carbon in laboratory and pilot-scale. In addition, auxiliary studies applying sociological research data will develop new approaches on risk communication with drinking water consumers. Using adjusted risk management instruments, a guide for drinking water and waste water businesses will be developed.

The entry and transport of antibiotics and the development of antibiotic in the urban waste water will be described by the research project ANTI-Resist. Therefore the sewage system of Dresden is inspected. A mass flow description is going to be based medical prescription data, transport data in the sewage system and release into surface waters. The analysis of prescription data and analysis of waste water/sediment samples will be integrated into a material flow model. The project aims to design suitable strategies for the reduction of antibiotics entry into urban waste water and to create an early warning and monitoring system. Project results will be communicated to experts and decision makers and will be integrated in training measures.

The amount of pollutants and pathogens released into sewage water system from hospitals is known to be an important issue, especially with regard to demographic change and rising life expectancy, as they are usually associated with increasing medication. Other than for hospitals the role of health care facilities is not well investigated up to now. The research project SAUBER+ aims to characterize the risk resulting from the emission of pharmaceuticals and pathogens from various health care facilities into the aquatic environment. Open questions exist with regard to the amount of active ingredients and the interaction of pathogens with medication residues building multi resistant germs. Furthermore an adaptable management strategy and a communication concept are missing.

Within a monitoring programme waste water of several health care facilities, nursing homes, hospices and clinics will be characterized and evaluated. In order to minimize the influence of pharmaceuticals and pathogens, the pretreatment of waste water streams will be optimized in a second step. Therefore a wide range of state-of-the-art technologies such as membrane bioreactor, ozonation, UV irradiation and activated carbon adsorption are considered. Their improvement and assessment together with operational strategies will lead to more efficient evaluation strategies and will ease their on-site implementation. Furthermore risk awareness in society will be strengthened in order to initiate changes in behavior patterns.

With regard to diffusive emission of micro pollutants and pathogens the research projects AGRO and RiskAGuA determine the occurrence and transport in rural areas.

The goal of the research project AGRO is to identify organic micropollutants and pathogens, their origin, distribution and mitigation in karst aquifers using microbial source tracking. A rural karst aquifer catchment with a single spring (Gallusquelle) located on the Swabian Alb in Southwestern Germany was chosen as investigation area. Monitoring data on the pollutant's behavior will provide the basis to develop a modeling tool to predict trace organics and pathogens concentrations at the Gallusquelle. With this instrument the distribution of waste water and manure concentrations can be predicted for different potential input-scenarios. In addition present indicator parameters (e.g. turbidity) were checked for the identification of the biological and chemical water quality as well as their suitability and characteristics in terms of risk management. The special characteristics of the Gallusquelle, i.a. as direct use as spring water for drinking water supply and diversified land use within the catchment area, allow a transfer of the project results to other aquifer systems.

The emission and distribution of veterinary pharmaceuticals and pathogenic microorganisms in the environment with a special attention of natural and artificial barriers to the development and spreading of resistancy via manure-utilization is in the focus of the research project RiskAGuA. To determine release and mass flow through various environmental compartments as natural 
barriers, solid waste from fat stock, dairy cattle and laying hen industry are investigated in lab scale column experiments. The impact of artificial barriers such as biogas-advanced waste water and waste treatment plants onto the spreading and persistence of veterinary pharmaceuticals, pathogenic microorganisms and their resistances is partly not well known. Therefore decentralized biotechnological processes are developed and optimized. Based on numerical modeling of groundwater flow and mass transfer processes in the unsaturated and saturated soil zone, the cause-effect correlations between pollutant emissions and effects on the water cycle can be determined. Together with the knowledge gained on the artificial barriers these results are implemented into an information platform. This ensures an internal and external knowledge transfer, e.g. as evidence-based action directives derived from experience and the communication of risk assessment and management strategies.

Besides its importance as source of drinking water supply, water acts as habitat for countless animals living in the water and the sediments. Against that background the emission of pollutants and pathogens out off final effluents is determined within the research projects TransRisk, SchussenAktivplus and Sichere Ruhr.

The characterization, communication and minimization of risks emanated from organic micropollutants and pathogens in the water cycle are in the focus of the research project TransRisk. For risk characterization organic micropollutants are classified in toxicological relevant contaminant categories with the aim to identiy indicator substances. The entity of metabolites which are of toxicological relevance for humans will be used for conveying a risk quotient. In addition, micropollutantinduced selection processes in bacteria and pathogens, which take place already at low, sub-inhibitory concentrations and lead to the formation of pathogenic factors (virulence, resistance), are integrated into the risk characterization. For minimization of risks the combination of biological and oxidative (ozonation) waste water treatment as well as the application of iron bacteria in biological waste water treatment will be compared with more established treatment techniques such as activated carbon filtration. To evaluate the risks of drinking water contamination by micropollutants and pathogens the developed concept will be implemented in the Donauried region (Southern Germany), considering the entire urban water cycle (waste water, surface water, groundwater as well as drinking water). The developed approaches for the communication of risks will be directly applied in the region.

The pollution of river Schussen situated in the southeast of Germany by micropollutants and potentially pathogenic bacteria is investigated within the research project SchussenAktivplus. Due to the highly populated catchment area and as tributary of Lake Constance (used for drinking water supply) the efficiency of water treatment technologies is important. Thus the project SchussenAktivplus focuses on technologies capable to reduce the release of micropollutants and potentially pathogenic bacteria from sewage treatment plants and rain overflow basins into surface waters. The effectiveness of applied technologies will be recorded by various biological in vitro and in vivo tests, e.g. reduction of toxic and endocrine potentials in effluents. Besides this, the ecosystem along the river Schussen will be supervised by effect analyses in different indigenous species including different fish species and benthic invertebrates relevant as food sources for fish. Finally, scientific studies will be accompanied by public relation, dissemination and communication of the results to relevant Stakeholder.

Beside drinking water supply the river Ruhr is a favoured destination for recreation, including swimming, even though there are microbiological risks and a bathing prohibition in place for most parts. Thus a hygienic evaluation is important especially with regard to the analytical proof of "new" trace substances in the Ruhr. To enable a swimming permission and to ensure the water quality for drinking water supply a comprehensive risk management concept is going to be developed and implemented within the research project Sichere Ruhr. To reduce microbial contaminations from urban drainage systems technical installations are already in place. Their success and influence on the environmental issues in the catchment area of the river Ruhr is monitored by establishing suitable rapid tests. Additionally a modelbased early warning system indicating the prohibition of bathing activities and the water quality is going to be invented.

\section{Competing interests}

The authors declare that they have no competing interests.

\section{Authors' contribution}

All authors have read and approved the final manuscript.

\section{Acknowledgements}

The scientific support project wants to acknowledge the Federal Ministry of Education and Research and Dr. Christina Jungfer, DECHEMA e.V., for her support with the article.

Received: 15 November 2012 Accepted: 15 November 2012 Published: 14 January 2013

doi:10.1186/2190-4715-25-1

Cite this article as: Huckele and Track: Risk management of emerging compounds and pathogens in the water cycle (RiSKWa) Environmental Sciences Europe 2013 25:1 